\title{
Criminologie
}

\section{La victimisation, la polyvictimisation et la délinquance chez les enfants et les jeunes adultes au Royaume-Uni}

\author{
Lorraine Radford, Susana Corral, Christine Bradley et Helen L. Fisher
}

Volume 47, numéro 1, printemps 2014

La polyvictimisation des jeunes

URI : https://id.erudit.org/iderudit/1024007ar

DOI : https://doi.org/10.7202/1024007ar

Aller au sommaire du numéro

Éditeur(s)

Les Presses de l’Université de Montréal

ISSN

0316-0041 (imprimé)

1492-1367 (numérique)

Découvrir la revue

Citer cet article

Radford, L., Corral, S., Bradley, C. \& Fisher, H. L. (2014). La victimisation, la polyvictimisation et la délinquance chez les enfants et les jeunes adultes au Royaume-Uni. Criminologie, 47(1), 59-83. https://doi.org/10.7202/1024007ar
Résumé de l'article

Cette étude explore l'impact et la nature de l'accumulation de victimisations sur la déviance autodéclarée des enfants et des jeunes adultes. Dans le cadre d'une enquête représentative menée auprès de ménages au Royaume-Uni en 2009, l'exposition à une gamme élargie d'expériences de victimisation ainsi que la délinquance autodéclarée ont été évaluées grâce à 4036 interviews auprès de participants de deux groupes : des enfants âgés de 11 à 17 ans et des jeunes adultes âgés de 18 à 24 ans. L'âge, le sexe (masculin) et le fait d'avoir vécu certaines formes de victimisation augmentaient considérablement les probabilités de polyvictimisation au cours de l'enfance. L'impact de la victimisation sur la délinquance variait selon le type de victimisation, le sexe et le groupe d'âge. La victimisation sexuelle durant l'enfance était fortement associée à la délinquance chez les femmes mais avait moins d'impact chez les hommes âgés de moins de 18 ans. La polyvictimisation avait le plus d'impact sur la délinquance des enfants âgés de 11 à 17 ans mais n'influençait pas significativement celle des jeunes femmes adultes. Les professionnels qui travaillent auprès des enfants et des jeunes doivent être à l'affût des concomitances et du caractère cumulatif de la victimisation durant l'enfance. Ils doivent également être outillés afin de repérer et d'aider ceux qui sont vulnérables à la polyvictimisation. 


\title{
La victimisation, la polyvictimisation et la délinquance chez les enfants et les jeunes adultes au Royaume-Uni ${ }^{1}$
}

\author{
Lorraine Radford ${ }^{2,3}$ \\ Professeure \\ University of Central Lancashire, Royaume-Uni \\ lradford@uclan.ac.uk \\ Susana Corral \\ Professeure \\ Universidad de Deusto, Espagne \\ susana.corral@deusto.es \\ Christine Bradley \\ Doctorante \\ University of Bristol, Royaume-Uni \\ ptycab@bristol.ac.uk \\ Helen L. Fisher \\ Professeure \\ King's College London, Royaume-Uni \\ helen.2.fisher@kcl.ac.uk
}

RÉSUMÉ - Cette étude explore l'impact et la nature de l'accumulation de victimisations sur la déviance autodéclarée des enfants et des jeunes adultes. Dans le cadre d'une enquête représentative menée auprès de ménages au Royaume-Uni en 2009, l'exposition à une gamme élargie d'expériences de victimisation ainsi que la délinquance autodéclarée ont été évaluées grâce à 4036 interviews auprès de participants de deux groupes: des enfants âgés de 11 à 17 ans et des jeunes adultes âgés de 18 à 24 ans. L'âge, le

1. Traduit de l'anglais par Juan Carlos Arellano López.

2. University of Central Lancashire, Harrington Building, School of Social Work, Preston, Lancashire, PR1 2HE, United Kindom.

3. Remerciements: cette recherche a été financée par la NSPCC, la Children's Charity, les British Home Stores et la TNS-BMRB. Helen L. Fisher a été soutenue par une bourse postdoctorale octroyée par le Medical Research Council and Economic and Social Research Council, au Royaume-Uni. Sherry Hamby, professeure à Sewanee: University of the South, États-Unis, a fourni des commentaires constructifs lors d'une ébauche préliminaire. 
sexe (masculin) et le fait d'avoir vécu certaines formes de victimisation augmentaient considérablement les probabilités de polyvictimisation au cours de l'enfance. L'impact de la victimisation sur la délinquance variait selon le type de victimisation, le sexe et le groupe d'âge. La victimisation sexuelle durant l'enfance était fortement associée à la délinquance chez les femmes mais avait moins d'impact chez les hommes âgés de moins de 18 ans. La polyvictimisation avait le plus d'impact sur la délinquance des enfants âgés de 11 à 17 ans mais n'influençait pas significativement celle des jeunes femmes adultes. Les professionnels qui travaillent auprès des enfants et des jeunes doivent être à l'affût des concomitances et du caractère cumulatif de la victimisation durant l'enfance. Ils doivent également être outillés afin de repérer et d'aider ceux qui sont vulnérables à la polyvictimisation.

MOTS-CLÉs • Polyvictimisation, délinquance, victimisation pendant l'enfance, maltraitance, Royaume-Uni.

\section{Introduction}

La maltraitance et la victimisation durant l'enfance ont lieu partout dans le monde. Elles contribuent à la mortalité des enfants en plus d'engendrer de lourdes conséquences sur la santé et sur le bien-être des enfants et des jeunes (Pinheiro, 2006). Ces conséquences incluent des effets durables sur la santé mentale, l'abus d'alcool et de drogues (particulièrement chez les filles), les comportements sexuels à risque, l'obésité et le comportement criminel, lequel peut perdurer jusqu'à l'âge adulte (Gilbert et al., 2008). Dans une étude réalisée auprès de 8000 adultes recrutés dans des centres de santé aux États-Unis, on a découvert une corrélation entre le nombre de formes de mauvais traitements déclaré et le score de santé mentale (Edwards et al., 2003). La maltraitance et les événements aversifs multiples sont d'ailleurs interreliés et produisent des effets cumulatifs sur la santé mentale des enfants (Donga et al., 2004). La recherche dans ce domaine reste cependant limitée par l'usage d'échantillons non représentatifs (p. ex., Green et al., 1999; Arata et al., 2007; Gustafsson et al., 2009) ou par la concentration sur quelques types de victimisation (p. ex., Bensley et al., 1999; Wolfe et al., 2001 et Kinard, 2004), ce qui empêche d'explorer l'impact relatif des formes de victimisation multiples ou spécifiques. De récents progrès en cette matière ont été rendus possibles par deux vastes études populationnelles aux États-Unis (la Developmental Victimization Survey [DVQ]: Finkelhor et al., 2005; et la National Survey of Children's Exposure to Violence [NatSCEV]: Finkelhor et al., 2009a), qui ont employé le Juvenile Victimization Questionnaire (JVQ) (Hamby et al., 2004a ; 2004b) couvrant ainsi un large éventail 
d'expériences de victimisation. Ces études ont démontré que l'exposition à différentes formes de victimisation et à d'autres événements aversifs provoquait des effets néfastes sur la santé mentale des enfants et des adolescents (Turner et al., 2006) mais que le fait de subir plusieurs types de victimisation (polyvictimisation) avait encore plus d'impact (Finkelhor et al., 2007a; 2007b). Or, puisque la prévalence et les contextes socioculturels diffèrent d'un pays à l'autre (Andrews et al., 2004), il importe de savoir si les résultats obtenus aux États-Unis s'appliquent ailleurs. Une étude européenne a examiné le lien entre la polyvictimisation et le fonctionnement psychologique des enfants (Gustafsson et al., 2009), mais celle-ci a été effectuée auprès d'un échantillon de commodité.

Le lien entre la maltraitance et la victimisation, notamment la victimisation multiple et la délinquance chez l'enfant, a suscité l'intérêt des criminologues (Cuevas et al., 2007; Burton et al., 2011 ;) afin d'identifier les enfants plus à risque d'adopter un comportement délinquant persistant durant toute leur vie (Egeland et al., 2002 ; Moffit et al., 2002; Wong et al., 2010). Spatz-Widom et Raskin-White (1997) ont constaté que les femmes maltraitées et négligées, contrairement aux hommes maltraités et négligés, sont plus à risque d'abuser de l'alcool ou d'autres drogues et de développer une dépendance, ou d'être arrêtées pour des crimes violents. L'interdépendance entre la maltraitance, la victimisation et la délinquance s'avère plutôt complexe. Par exemple, bien que les enfants maltraités et victimisés puissent être plus à risque d'adopter un comportement délinquant, il existe d'autres facteurs qui influencent la délinquance des jeunes et ceux-ci peuvent devenir délinquants sans une expérience de maltraitance préalable (Cuevas et al. 2007). Une explication partielle du lien entre la délinquance et la victimisation relève du fait que les jeunes maltraités sont davantage enclins à s'engager dans des comportements à risque et d'être ainsi exposés à de plus grands risques de victimisation (Cuevas et al., 2007). Cette idée est corroborée par une étude prospective menée auprès de filles aux États-Unis par Wilson et Spatz-Widom (2008) qui ont observé que celles qui sont maltraitées sont plus susceptibles d'avoir un contact sexuel avant l'âge de 15 ans, de se prostituer jeunes adultes et de contracter le VIH au milieu de l'âge adulte. Il est possible que la victimisation ait un impact différent sur la délinquance des filles et des garçons.

Finkelhor et al. (2009b) ont identifié quatre trajectoires menant à la polyvictimisation: a) habiter dans une collectivité dangereuse, où les 
risques de victimisation sont plus importants; b) vivre dans un milieu familial dangereux, ce qui augmente la vulnérabilité à d'autres genres de mauvais traitements; $c$ ) provenir d'un milieu familial chaotique à problèmes multiples, dans lequel le manque de supervision parentale entraîne un risque pour l'enfant; et d) éprouver des problèmes émotionnels qui accroissent les comportements à risque, génèrent l'antagonisme et affaiblissent la capacité de s'autoprotéger. En outre, il a été découvert que les premières manifestations de la polyvictimisation étaient particulièrement susceptibles de survenir au cours de l'année précédant les $7^{\mathrm{e}}$ et $15^{\mathrm{e}}$ anniversaires de l'enfant, correspondant, à peu près, à l'entrée à l'école primaire puis secondaire. L'identification de telles trajectoires ainsi que des périodes du développement associées à un risque accru devrait aider les intervenants à créer des programmes de prévention de la polyvictimisation chez les enfants vulnérables.

Dans cet article, nous cherchons à poursuivre les travaux concernant l'impact de l'accumulation de victimisations sur la déviance autodéclarée des enfants, des adolescents et des jeunes adultes i) au RoyaumeUni; ii) incluant un large spectre d'expériences de victimisation; iii) en nous servant de définitions, par groupe d'âge, de la polyvictimisation; et iv) en ayant recours à un échantillon national représentatif des enfants, adolescents et jeunes adultes. Deux questions guident la recherche: quelles sont les caractéristiques des jeunes polyvictimisés appartenant à un échantillon de la population du Royaume-Uni? Tenant compte d'autres facteurs de risque connus, quel est l'impact de différents types de victimisation et de la polyvictimisation sur les niveaux de délinquance déclarée par les garçons et les filles?

\section{Méthodologie}

L'analyse est basée sur les données tirées de 4036 interviews menées auprès de ménages sélectionnés de façon aléatoire, dont les participants formaient deux groupes: 2275 enfants âgés de 11 à 17 ans, et 1761 jeunes adultes âgés de 18 à 24 ans. Les entrevues, réalisées entre mars et décembre 2009, faisaient partie d'une plus ample étude britannique sur la maltraitance et autres sortes de victimisation subies par les enfants et les jeunes. La méthodologie a été publiée ailleurs (Radford et al., 2011; Radford et al., 2013) et, par conséquent, seule une brève description en sera faite ici. Une firme spécialisée en recherche sociale, TNS-BMRB, a été chargée de réaliser les interviews porte à porte. En 
se basant sur une pratique commune pour mener les enquêtes sur la victimisation au Royaume-Uni, dont la British Crime Survey (Chaplin, Flatley \& Smith, 2011), l'auto-interview assistée par ordinateur (CASI) et le CASI audio ont été employées. À la suite de l'envoi de lettres de préavis, des entrevues face à face ont eu lieu avec les répondants, dans leur ménage. Parmi les enfants et les jeunes adultes compris dans l'enquête, $51,9 \%$ étaient des femmes $(\mathrm{N}=2094)$ et $48,1 \%$ étaient des hommes $(\mathrm{N}=1942)$; il y avait $84,7 \%$ de Britanniques blancs, $2,9 \%$ de Blancs (autres), 2,4\% d'ethnicité mixte, 5,5\% de Sud-Asiatiques, $2,4 \%$ de Britanniques noirs, d'Africains ou d'Afro-Antillais, et 2,2\% de Chinois ou autres groupes ethniques. Le taux de réponse global de l'enquête se situait à $60,4 \%$. Au total, 12,9\% des jeunes âgés de 11 à 17 ans et $13,5 \%$ des jeunes adultes âgés de 18 à 24 ans affirmaient souffrir d'une quelconque forme d'incapacité.

En ce qui concerne les jeunes âgés de 11 à 17 ans, leur principal tuteur (tel que défini par les parents eux-mêmes) a été interrogé en premier sur des sujets généraux concernant la famille. Les jeunes réalisaient alors l'entrevue assistée par ordinateur portant sur les expériences de victimisation. Les intervieweurs devaient s'assurer que le jeune pouvait répondre aux questions de l'entretien sans être surveillé par une personne du ménage. Simultanément, le tuteur recevait un questionnaire papier à remplir. Les jeunes adultes répondaient seuls à l'ensemble de l'interview, incluant les questions sur leur passé familial durant l'enfance.

Afin d'évaluer l'exposition à un large éventail de maltraitance et de victimisation, une version modifiée du Juvenile Victimization Questionnaire (JVQ) (Hamby et al., 2004a ; 2004b) a été employée. Le JVQ se compose de 35 questions réparties en cinq modules: crime conventionnel; maltraitance; victimisation commise par un pair ou la fratrie; victimisation sexuelle; le fait d'être témoin de victimisation ou de victimisation indirecte (les questions s'adressaient au groupe d'âge de 11 à 17 ans; la référence de temps a été ajustée pour le groupe d'âge de 18 à 24 ans, les questions étant posées rétrospectivement). Des questions de suivi ont été posées afin de savoir si la victimisation avait été subie au cours des 12 mois précédents (non applicable au groupe de 18 à 24 ans), à quelle fréquence cette expérience s'était produite, qui était l'agresseur et si la victime interprétait son expérience comme étant abusive ou violente. Deux questions ont été ajoutées (connues sous l'abréviation NSPCC ou National Society for the Prevention of Cruelty to Children): l'une portant sur la violence sexuelle exercée par un adulte 
en position de confiance (à l'intention des jeunes âgés de 16 et 17 ans), l'autre se penchant sur le fait de bousculer ou de secouer un enfant. La négligence a été évaluée à l'aide d'une question du JVQ et de 13 questions spécifiques en fonction du groupe d'âge, suivant les conseils du gouvernement britannique (Gouvernement britannique, 2010). Pour les participants d'âge majeur, la négligence a été évaluée par des questions utilisées antérieurement lors de l'enquête menée par la NSPCC (Cawson et al., 2000).

Une version adaptée de la mesure d'événements aversifs sans victimisation, décrite par Turner et al. (2006), a été employée pour évaluer les traumatismes non violents et les facteurs de stress chroniques subis par les participants au cours de leur enfance. Elle est composée de neuf questions relatives aux accidents, aux maladies graves, aux décès, à l'itinérance, à la consommation abusive d'alcool ou de drogues parmi les membres de la famille, à la séparation des parents et à l'emprisonnement (plus de détails dans Radford et al., 2011).

Afin d'évaluer la délinquance, un instrument d'autoévaluation de délinquance juvénile comportant 14 questions a été utilisé auprès des participants. Ces questions étaient fondées sur la mesure de Cuevas et al. (2007).

\section{Enjeux éthiques}

La recherche a été approuvée par le Comité d'éthique de la recherche de la NSPCC et elle a bénéficié d'un examen éthique continuel de la part d'experts internationaux durant toute la collecte de données. Un consentement écrit des parents était requis pour toute personne âgée de moins de 18 ans. Ainsi, pour les participants âgés de 11 à 17 ans, le consentement était accordé par le jeune et reconfirmé à l'écran au début de l'interview assistée par ordinateur (Radford \& Ellis, 2012). Un système permettant d'identifier les enfants susceptibles de danger immédiat a été conçu; un protocole pour le renvoi à la protection de l'enfance a été adopté; et un système de soutien indépendant a été instauré avec l'aide de la ChildLine (services téléphoniques et en ligne offerts par la NSPCC), de la ligne d'aide de la NSPCC (service de conseil pour les professionnels et pour tous ceux qui se préoccupent de la sécurité et du bien-être des enfants) ainsi que d'un conseiller indépendant. Durant l'interview, les participants pouvaient indiquer s'ils désiraient recevoir de l'aide ou parler de leurs expériences avec quel- 
qu'un. À la fin de l'entrevue, tous les participants se voyaient offrir un «debriefing» de la rencontre, de même que de l'information sur les services de soutien offerts.

\section{Analyse}

Des facteurs de pondération ont été appliqués à l'ensemble des analyses, afin de compenser les probabilités inégales d'échantillonnage et les réponses inégales selon le groupe d'âge, le sexe, le fait d'être propriétaire ou locataire, la situation professionnelle, le groupe ethnique et la région d'origine. Les analyses ont été effectuées séparément pour les deux groupes d'âge mentionnés. Des catégories ont été créées à partir des éléments du JVQ et des questions de la NSPCC pour faire état des différents types de victimisation pendant l'enfance au cours des 12 derniers mois (pour le groupe de jeunes âgés de 11 à 17 ans) et au cours de leur enfance entière (pour les deux groupes d'âge). Ces catégories comprennent la maltraitance, la négligence, les mauvais traitements psychologiques, la violence physique, la victimisation sexuelle, l'exposition à la violence familiale et le fait d'être témoin de violence dans la communauté. D'autres catégories ont également été créées afin d'illustrer la victimisation selon les différents types d'agresseurs. On compte parmi ceux-ci:

- La maltraitance perpétrée par les parents ou par les tuteurs (incluant toute violence physique, psychologique ou sexuelle, ainsi que toute négligence commise par le parent, le tuteur ou leurs conjoints, mais excluant l'exposition à la violence familiale et parentale qui est évaluée séparément);

- La maltraitance de la part d'adultes qui n'habitent pas le foyer familial (toute violence physique, psychologique ou sexuelle commise sur l'enfant par un adulte autre que son parent, son tuteur ou leurs conjoints);

- La victimisation de la part d'un pair (toute violence physique ou psychologique et toute victimisation sexuelle faites par tout jeune de moins de 18 ans, excluant la fratrie et le partenaire intime du jeune victimisé);

- La victimisation commise par la fratrie (toute violence physique et psychologique, et toute victimisation sexuelle de la part d'un frère ou une sœur de moins de 18 ans); 
- La victimisation d'un jeune par son partenaire intime (toute violence physique et psychologique, et toute victimisation sexuelle infligées par le partenaire intime, peu importe son âge).

Les catégories suivantes ont également été tirées du JVQ, dans le but d'évaluer les taux de polyvictimisation (PV) dans les deux groupes d'âge de l'étude:

- Une variable continue basée sur la somme des divers types de victimisation vécus durant l'enfance;

- Une variable dichotomique visant à mesurer les cas de «polyvictimisation élevée» (où 0 indique les cas n'étant pas de la "polyvictimisation élevée» et 1 indique les cas de polyvictimisation élevée). Les cas de "polyvictimisation élevée» correspondent au 10\% de polyvictimes ayant vécu le plus grand nombre d'expériences de victimisation distinctes dans chacun des groupes d'âge, 13 et plus chez les 11 à 17 ans et 15 et plus chez les 18 à 24 ans en ce qui a trait à la victimisation au cours de toute l'enfance; 5 et plus chez les 11 à 17 ans pour les victimisations vécues au cours des 12 derniers mois.

Pour simplifier la présentation des résultats et faire preuve de cohérence avec les autres recherches publiées (Finkelhor et al., 2007a; 2007b), les symptômes traumatiques ont été calculés en sommant les réponses de chaque jeune et jeune adulte au Trauma Symptom Checklist for Children (TSCC) et au Trauma Symptom Checklist (TSC), respectivement. Ces cotes totales ont été standardisées à partir de la moyenne et de l'écart-type du groupe d'âge correspondant et ont ensuite été réunies afin d'établir une cote globale du traumatisme permettant la comparaison entre les participants des différents groupes d'âge. Les cotes de délinquance ont été combinées dans le but de créer une cote de délinquance globale.

Des régressions logistiques multiples de type hiérarchique ont été utilisées pour tester les variables qui augmentaient les probabilités qu'un jeune âgé de 11 à 17 ans et qu'un jeune adulte aient vécu de la polyvictimisation élevée durant l'enfance. Des régressions linéaires multiples de type hiérarchique ont aussi été utilisées pour évaluer l'impact de différents types de victimisation et de la polyvictimisation sur les cotes de délinquance autodéclarée. 


\section{Résultats}

Prévalence des formes de victimisation et de polyvictimisation

Le tableau 1 présente les taux des divers types de victimisation perpétrés par différents types d'agresseurs au cours de toute l'enfance et au cours des 12 mois précédents.

Les filles étaient plus nombreuses que les garçons à rapporter n'avoir subi aucune victimisation ou à en avoir subi une seule forme (Tableau 2). En effet, tel que l'ont constaté Finkelhor et al. (2007a), les garçons présentaient un nombre moyen de victimisation plus élevé durant l'ensemble de leur vie et au cours des 12 derniers mois et un taux de polyvictimisation légèrement plus élevé que les filles.

\section{Facteurs de risque liés à la polyvictimisation}

Les jeunes et jeunes adultes polyvictimisés ont vécu des niveaux particulièrement élevés de certains types de victimisation durant leur enfance, notamment l'exposition à la violence dans la communauté, la victimisation par les pairs, la victimisation sexuelle, la maltraitance commise par un parent et la violence familiale (graphique 1). Il importe de mentionner qu'un jeune polyvictimisé sur cinq (c'est-à-dire $21,2 \%$, âgés de 11 à 17 ans) et qu'un jeune adulte polyvictimisé sur trois (à savoir 34,7\%, âgés de 18 à 24 ans) montraient un certain degré d'incapacité.

Des régressions logistiques multiples de type hiérarchique ont été menées afin de tester les variables qui augmentaient les probabilités de polyvictimisation chez les jeunes et jeunes adultes. Durant l'étape 1, les variables, identifiées comme des risques probables dans d'autres études (Farrington et al., 2006; Finkelhor et al., 2007a; 2007b), ont été insérées en tant que covariables afin de calculer les rapports de cotes (odds ratios) de polyvictimisation élevée durant l'enfance: l'âge du jeune au moment de l'interview; le sexe; l'origine ethnique; une quelconque incapacité; le fait de vivre actuellement avec ses deux parents biologiques ou, si adulte, durant la majeure partie de son enfance; d'autres adversités sans victimisation; l'exposition à la violence dans la communauté; et le groupe professionnel des parents (la situation professionnelle des parents la plus élevée au moment de l'entrevue, comme définie par l'échelle de classement social de la 
TABLEA U 1

Fréquence des cas de victimisation durant toute l'enfance (TE) et au cours de la dernière année (DA) selon l'agresseur, le type de victimisation, le groupe d'âge et le sexe de la victime (résultats pondérés, $95 \%$ d'intervalle de confiance)

\begin{tabular}{|c|c|c|c|c|c|c|c|c|c|}
\hline \multirow{3}{*}{ Type de victimisation } & \multicolumn{6}{|c|}{ 11-17 ans } & \multicolumn{3}{|c|}{$18-24$ ans } \\
\hline & \multicolumn{3}{|c|}{ TE } & \multicolumn{3}{|c|}{ DA } & \multicolumn{3}{|c|}{ TE } \\
\hline & Tous & Garçons & Filles & Tous & Garçons & Filles & Tous & Garçons & Filles \\
\hline Exposition à la violence familiale & $\begin{array}{r}17,5 \% \\
(302) \\
+/-1,1^{a}\end{array}$ & $\begin{array}{r}16,4 \% \\
(145)\end{array}$ & $\begin{array}{r}18,7 \% \\
(157)\end{array}$ & $\begin{array}{r}2,5 \% \\
(43) \\
+-0,6\end{array}$ & $\begin{array}{r}2,1 \% \\
(19)\end{array}$ & $\begin{array}{r}2,9 \% \\
(25)\end{array}$ & $\begin{array}{r}23,7 \% \\
(449) \\
+/-2\end{array}$ & $\begin{array}{r}19,5 \% \\
(188)\end{array}$ & $\begin{array}{r}28,0 \% \\
(260)\end{array}$ \\
\hline Enfant maltraité par un parent ou un tuteurb & $\begin{array}{r}21,9 \% \\
(379) \\
+/-1,7\end{array}$ & $\begin{array}{r}22,7 \% \\
(201)\end{array}$ & $\begin{array}{r}21,2 \% \\
(178)\end{array}$ & $\begin{array}{r}6,0 \% \\
(103) \\
+/-1\end{array}$ & $\begin{array}{r}5,7 \% \\
(51)\end{array}$ & $\begin{array}{r}6,2 \% \\
(52)\end{array}$ & $\begin{array}{r}24,5 \% \\
(465) \\
+/-2\end{array}$ & $\begin{array}{r}22,7 \% \\
(219)\end{array}$ & $\begin{array}{r}26,5 \% \\
(246)\end{array}$ \\
\hline $\begin{array}{l}\text { Victimisation sexuelle perpétrée par adulte ou } \\
\text { pair }\end{array}$ & $\begin{array}{r}16,5 \% \\
(285) \\
+/-1,5\end{array}$ & $\begin{array}{r}12,5 \% \\
(111)\end{array}$ & $\begin{array}{r}20,8 \% \\
(175)\end{array}$ & $\begin{array}{r}9,4 \% \\
(163) \\
+/-1,2\end{array}$ & $\begin{array}{r}6,8 \% \\
(60)\end{array}$ & $\begin{array}{r}12,2 \% \\
(102)\end{array}$ & $\begin{array}{r}24,1 \% \\
(456) \\
+/-2\end{array}$ & $\begin{array}{r}17,4 \% \\
(168)\end{array}$ & $\begin{array}{r}31,0 \% \\
(288)\end{array}$ \\
\hline Victimisation par les pairs ${ }^{c}$ & $\begin{array}{r}59,5 \% \\
(1028) \\
+/-2\end{array}$ & $\begin{array}{r}66,0 \% \\
(585)\end{array}$ & $\begin{array}{r}52,7 \% \\
(443)\end{array}$ & $\begin{array}{r}35,3 \% \\
(609) \\
+/-2\end{array}$ & $\begin{array}{r}41,2 \% \\
(365)\end{array}$ & $\begin{array}{r}29,1 \% \\
(244)\end{array}$ & $\begin{array}{l}63,2 \% \\
(1198) \\
+/-2,3\end{array}$ & $\begin{array}{r}69,6 \% \\
(671)\end{array}$ & $\begin{array}{r}56,6 \% \\
(526)\end{array}$ \\
\hline Victimisation par la fratrie ${ }^{d}$ & $\begin{array}{r}31,8 \% \\
(550) \\
+/-1,9\end{array}$ & $\begin{array}{r}29,3 \% \\
(259)\end{array}$ & $\begin{array}{r}34,6 \% \\
(290)\end{array}$ & $\begin{array}{r}16,0 \% \\
(275) \\
+/-1,5\end{array}$ & $\begin{array}{r}15,8 \% \\
(140)\end{array}$ & $\begin{array}{r}16,1 \% \\
(135)\end{array}$ & $\begin{array}{r}25,2 \% \\
(478) \\
+/-2\end{array}$ & $\begin{array}{r}23,4 \% \\
(225)\end{array}$ & $\begin{array}{r}27,2 \% \\
(253)\end{array}$ \\
\hline Victimisation commise par un partenaire intime ${ }^{\mathrm{e}}$ & $\begin{array}{r}7,9 \% \\
(137) \\
+/-1,1\end{array}$ & $\begin{array}{r}7,0 \% \\
(62)\end{array}$ & $\begin{array}{r}8,9 \% \\
(74)\end{array}$ & $\begin{array}{r}5,0 \% \\
(86) \\
+/-0,9\end{array}$ & $\begin{array}{r}4,2 \% \\
(37)\end{array}$ & $\begin{array}{r}5,8 \% \\
(49)\end{array}$ & $\begin{array}{r}13,4 \% \\
(254) \\
+/-1,6\end{array}$ & $\begin{array}{r}10,7 \% \\
(103)\end{array}$ & $\begin{array}{r}16,2 \% \\
(150)\end{array}$ \\
\hline
\end{tabular}


TABLE 1 (suite)

\begin{tabular}{|c|c|c|c|c|c|c|c|c|c|}
\hline \multirow{3}{*}{ Type de victimisation } & \multicolumn{6}{|c|}{$11-17$ ans } & \multirow{2}{*}{\multicolumn{3}{|c|}{$\frac{18-24 \text { ans }}{T E}$}} \\
\hline & \multicolumn{3}{|c|}{ TE } & \multicolumn{3}{|c|}{ DA } & & & \\
\hline & Tous & Garçons & Filles & Tous & Garçons & Filles & Tous & Garçons & Filles \\
\hline Exposition à la violence dans la communauté & $\begin{array}{r}61,4 \% \\
(1060) \\
+/-2\end{array}$ & $\begin{array}{r}67,9 \% \\
(601)\end{array}$ & $\begin{array}{r}54,6 \% \\
(459)\end{array}$ & $\begin{array}{r}31,2 \% \\
(539) \\
+/-1,9\end{array}$ & $\begin{array}{r}34,0 \% \\
(301)\end{array}$ & $\begin{array}{r}28,3 \% \\
(238)\end{array}$ & $\begin{array}{l}66,5 \% \\
(1259) \\
+/-2,2\end{array}$ & $\begin{array}{r}73,0 \% \\
(705)\end{array}$ & $\begin{array}{r}59,7 \% \\
(555)\end{array}$ \\
\hline
\end{tabular}

a Intervalle de confiance.

Toute violence physique, psychologique ou sexuelle, et toute négligence de l'enfant de la part d'un parent ou d'un tuteur, excluant l'exposition à la violence familiale parentale.

Toute violence physique ou psychologique, et toute victimisation sexuelle de l'enfant par une personne âgée de moins de 18 ans, excluant la victimisation perpétrée par le partenaire intime ou la fratrie.

Toute violence physique ou psychologique, et toute victimisation sexuelle de l'enfant perpétrées par un frère ou une sœur.

Toute violence physique ou psychologique, et toute victimisation sexuelle commises par le partenaire intime âgé de plus ou de moins 18 ans.

Note: tous les pourcentages sont les pourcentages (pondérés) des enfants et des jeunes appartenant au groupe d'âge ayant subi ce type de victimisation. Les chiffres entre

parentheses indiquent le nombre d'individus que representent les pourcentages. 
TABLEAU 2

Cas de polyvictimisation durant toute l'enfance (TE) et au cours de la dernière année (DA) selon le groupe d'âge et le sexe de la victime

\begin{tabular}{|c|c|c|c|c|c|c|c|c|c|}
\hline \multirow[t]{2}{*}{ Nombre de cas de victimisation } & \multicolumn{3}{|c|}{$\begin{array}{c}\text { 11-17 ans } \\
\text { TE }\end{array}$} & \multicolumn{3}{|c|}{$\begin{array}{c}\text { 11-17 ans } \\
\text { DA }\end{array}$} & \multicolumn{3}{|c|}{$\begin{array}{c}18-24 \text { ans } \\
\text { TE }\end{array}$} \\
\hline & Tous & Garçons & Filles & Tous & Garçons & Filles & Tous & Garçons & Filles \\
\hline Aucun & $16,3 \%$ & $12,2 \%$ & $20,6 \%$ & $43,3 \%$ & $39,0 \%$ & $48,1 \%$ & $12,7 \%$ & $11,1 \%$ & $14,3 \%$ \\
\hline Un & $11,6 \%$ & $10,6 \%$ & $33,4 \%$ & $18,9 \%$ & $18,0 \%$ & $20,0 \%$ & $10,9 \%$ & $8,8 \%$ & $13,0 \%$ \\
\hline Plusieurs & $63,6 \%$ a & $68,4 \%$ a & $37,9 \%$ & $26,6 \%{ }^{c}$ & $30,8 \% c$ & $21,9 \%{ }^{c}$ & $67,6 \% \mathrm{e}^{\mathrm{e}}$ & $70,5 \% \mathrm{e}^{\mathrm{e}}$ & $64,7 \%{ }^{\mathrm{e}}$ \\
\hline Polyvictimisation & $8,5 \%$ b & $8,8 \%$ b & $8,1 \%{ }^{b}$ & $11,2 \%{ }^{d}$ & $12,2 \%{ }^{d}$ & $10,0 \%{ }^{d}$ & $8,8 \%^{f}$ & $9,6 \%^{f}$ & $8,1 \%^{f}$ \\
\hline Nombre moyen de victimisations & 5,2 & 5,6 & 4,8 & 1,7 & 1,9 & 1,6 & 6,3 & 6,8 & 5,7 \\
\hline
\end{tabular}

2 à 13 victimisations.

13 victimisations ou plus.

2 à 5 victimisations.

5 victimisations ou plus.

2 à 15 victimisations.

15 victimisations ou plus. 
GRAPHIQUE 1

Pourcentage de garçons et de filles polyvictimes durant toute leur enfance et au cours des 12 derniers mois en lien avec certains types de victimisation

11-17 ans depuis l'enfance

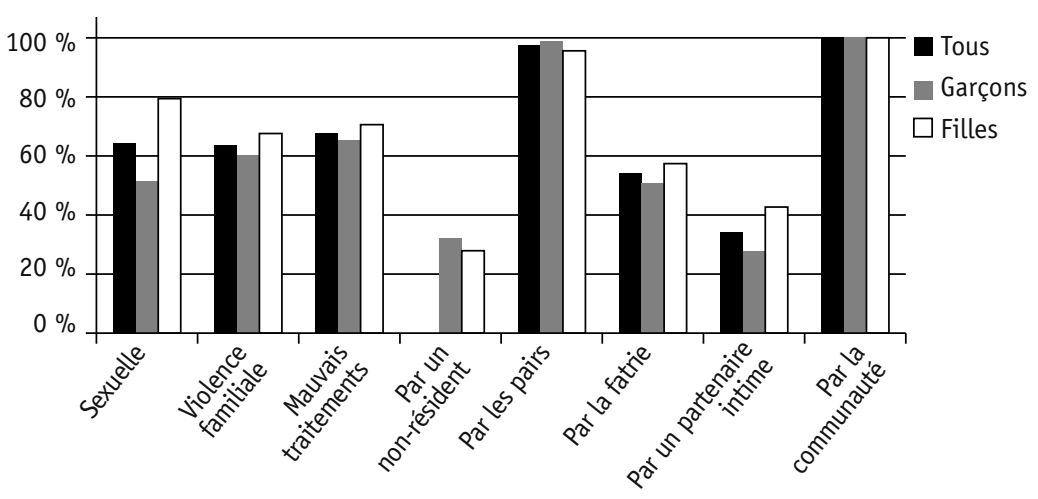

11-17 ans depuis la dernière année

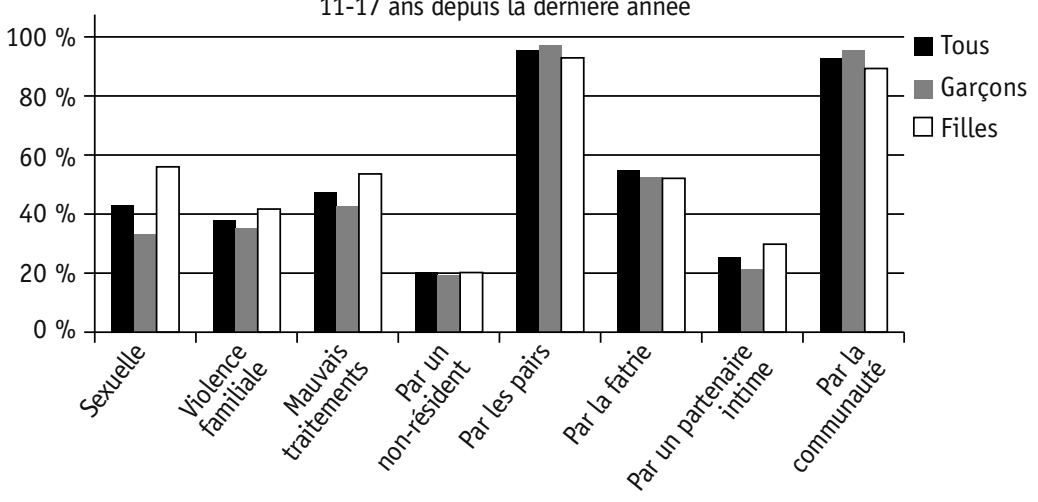

18-24 ans dans l'enfance

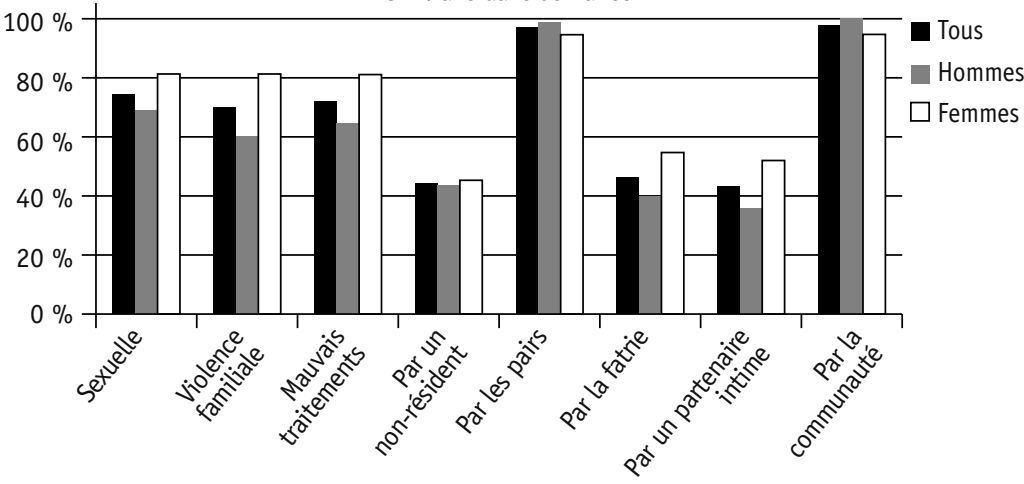


British National Readership Survey ${ }^{4}$ ). Lors de l'étape 2, les covariables suivantes ont été ajoutées: toute expérience de victimisation perpétrée par un pair, un frère ou une sœur, un partenaire intime ou un adulte ne résidant pas avec l'enfant; la maltraitance commise par un parent ou tuteur; l'exposition à la violence familiale parentale; et toute expérience de victimisation sexuelle durant l'enfance.

Pour les deux groupes d'âge, le fait d'être un garçon et de subir certains types de victimisation augmentait significativement les probabilités de polyvictimisation durant toute l'enfance (la victimisation commise par la fratrie et par un partenaire intime était significative uniquement chez les jeunes adultes). Bien qu'un enfant polyvictimisé sur cinq était atteint d'une quelconque incapacité, cette caractéristique était uniquement associée aux probabilités accrues de polyvictimisation chez les jeunes adultes interrogés (Tableau 3). La maltraitance commise par un parent/tuteur et la victimisation de la part des pairs augmentaient significativement les probabilités qu'un jeune ait été polyvictimisé au cours des 12 derniers mois.

\section{Polyvictimisation et délinquance}

Ce sont $42,3 \%$ des jeunes de 11 à 17 ans $(37,5 \%$ des garçons et $47,3 \%$ des filles) et $17,3 \%$ des jeunes adultes (16,8\% des hommes et $17,7 \%$ des femmes) qui ont déclaré n'avoir commis aucun acte de délinquance durant l'enfance. Or, nous savons que les taux de délinquance tendent à être relativement bas à 11 ans et augmentent à l'adolescence. Nos résultats indiquent d'ailleurs que les jeunes âgés de 11 à 17 ans rapportaient moins de comportements délinquants que les jeunes adultes. Le graphique 2 montre que les taux moyens de délinquance autodéclarée, ainsi que les taux élevés de délinquance (ceux situés au décile supérieur) augmentaient de façon stable et divergeaient légèrement selon le sexe à partir de 12 ans, atteignant leur sommet à 17 ans.

4. $\mathrm{AB}=$ postes administratifs, professionnels ou de gestion de niveau élevé ou intermédiaire. $\mathrm{C} 1$ = postes administratifs, professionnels ou de gestion de niveau débutant, de supervision ou de bureau. $\mathrm{C} 2=$ travail manuel spécialisé. $\mathrm{DE}=$ travail manuel semispécialisé et non qualifié; prestataires d'une pension de retraite; employés occasionnels ou peu qualifiés; ou sans emploi et bénéficiaires de prestations de chômage par l'État seulement. 
TABLEAU 3

\begin{abstract}
Variables associées au risque de polyvictimisation élevée durant toute l'enfance (TE) ou au cours de la dernière année (DA)
\end{abstract}

\begin{tabular}{|c|c|c|c|}
\hline Variable & $\begin{array}{c}\text { De } 11 \text { à } 17 \text { ans } \\
\text { (TE) } \\
\text { Rapport } \\
\text { de cotes de } \\
\text { polyvictimisation }\end{array}$ & $\begin{array}{c}\text { De } 11 \text { à } 17 \text { ans } \\
\text { (DA) } \\
\text { Rapport } \\
\text { de cotes de } \\
\text { polyvictimisation }\end{array}$ & $\begin{array}{c}\text { De } 18 \text { à } 24 \text { ans } \\
\text { (TE) } \\
\text { Rapport } \\
\text { de cotes de } \\
\text { polyvictimisation }\end{array}$ \\
\hline Sexe (garçon) & 2,6 ** & $\mathrm{ns}^{\mathrm{a}}$ & 2,6 ** \\
\hline Incapacité & ns & ns & $3,1^{* *}$ \\
\hline $\begin{array}{l}\text { Autres adversités } \\
\text { subies durant } \\
\text { l'enfance }\end{array}$ & ns & ns & $1,5^{* * *}$ \\
\hline $\begin{array}{l}\text { Victimisation } \\
\text { sexuelle }\end{array}$ & $4,6^{* * *}$ & ns & $4,6 * * *$ \\
\hline $\begin{array}{l}\text { Exposition à la } \\
\text { violence familiale } \\
\text { parentale }\end{array}$ & $4,5^{* * *}$ & ns & $2,4^{* *}$ \\
\hline $\begin{array}{l}\text { Maltraitance } \\
\text { commise par un } \\
\text { parent/tuteur }\end{array}$ & $2,5^{* *}$ & 1,9 ** & $3,5^{* * *}$ \\
\hline $\begin{array}{l}\text { Maltraitance } \\
\text { commise par un } \\
\text { adulte ne résidant } \\
\text { pas au domicile }\end{array}$ & $3,3^{* *}$ & ns & $2,0^{*}$ \\
\hline $\begin{array}{l}\text { Victimisation } \\
\text { commise par } \\
\text { la fratrie }\end{array}$ & ns & ns & $1,8^{*}$ \\
\hline $\begin{array}{l}\text { Victimisation } \\
\text { commise par } \\
\text { un pair }\end{array}$ & $13,8^{*}$ & $7,2^{*}$ & $4,7^{*}$ \\
\hline $\begin{array}{l}\text { Victimisation } \\
\text { commise par un } \\
\text { partenaire intime }\end{array}$ & ns & ns & $3,1^{* *}$ \\
\hline
\end{tabular}

a non significatif.

${ }^{*} p<0,05 ;{ }^{* *} p<0,01 ;{ }^{* *} p<0,001$.

Des régressions linéaires hiérarchiques ont été réalisées pour savoir si la polyvictimisation durant l'enfance avait un plus grand impact sur la délinquance autodéclarée que d'autres formes de victimisation subies. À l'étape $1 \mathrm{du}$ modèle de régression, des facteurs de risque démographiques liés à la délinquance ont été insérés: l'âge de l'enfant; l'origine ethnique; le groupe professionnel des parents; l'expérience d'autres types d'adversité durant l'enfance; et le fait de vivre séparé d'un parent 
GRAPHIQUE 2

Taux moyens de délinquance autodéclarée et taux élevé de délinquance (décile supérieur) des garçons et des filles selon l'âge

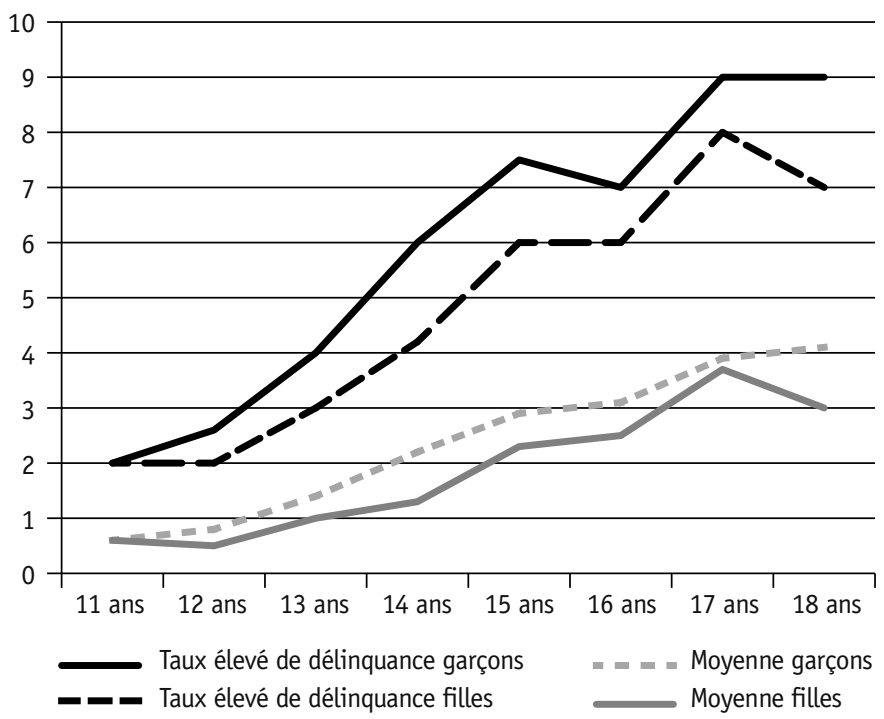

biologique. Lors de l'étape 2, les différentes formes de victimisation durant l'enfance ont été ajoutées: la victimisation sexuelle; la victimisation commise par un pair, la fratrie ou un partenaire intime; la maltraitance faite par un parent/tuteur; l'exposition à la violence familiale parentale; la maltraitance faite par un adulte ne résidant pas avec l'enfant; et l'exposition à la violence dans la communauté. Enfin, à l'étape 3, la polyvictimisation a été ajoutée, soit le fait d'avoir subi 13 (chez les 11 à 17 ans) ou 15 (chez les 18 à 24 ans) formes de victimisation distinctes ou plus durant l'enfance. Le tableau 4 indique, tant pour les garçons que pour les filles des deux groupes d'âge, les changements quant à la validité d'ajustement ("goodness of fit statistics») du modèle de régression linéaire pour chacune des trois étapes, les coefficients standardisés $(\beta)$, ainsi que le seuil de signification des variables insérées à l'étape 2 (sans polyvictimisation [-PV]) et à l'étape 3 (avec polyvictimisation [+PV]).

Le tableau 4 démontre que l'adversité sans victimisation exerce un impact particulièrement profond sur la délinquance autodéclarée, et ce, pour les deux sexes et groupes d'âge. Par rapport aux facteurs de risque démographiques, quelques différences entre les sexes et les groupes 
TA B LEA U 4

Analyse de régression hiérarchique de la cote de délinquance autodéclarée des garçons et filles de 11 à 17 ans et de 18 à 24 ans selon les facteurs de risque démographiques, les différents types de victimisation et la polyvictimisation élevée

\begin{tabular}{|c|c|c|c|c|c|c|c|c|}
\hline & \multicolumn{4}{|c|}{11 à 17 ans } & \multicolumn{4}{|c|}{18 à 24 ans } \\
\hline & \multicolumn{2}{|c|}{ Garçons } & \multicolumn{2}{|c|}{ Filles } & \multicolumn{2}{|c|}{ Garçons } & \multicolumn{2}{|c|}{ Filles } \\
\hline \multicolumn{9}{|c|}{ Statistiques de validité de l'ajustement } \\
\hline Étape $1^{\mathrm{a}}$ & \multicolumn{2}{|c|}{$\begin{array}{l}\mathrm{R}^{2} \text { ajusté }=, 301 \\
\text { Variation } \mathrm{R}^{2}=, 308^{* * *}\end{array}$} & \multicolumn{2}{|c|}{$\begin{array}{l}\mathrm{R}^{2} \text { ajusté }=, 342 \\
\text { Variation } \mathrm{R}^{2}=, 348^{* * *}\end{array}$} & \multicolumn{2}{|c|}{$\begin{array}{l}\mathrm{R}^{2} \text { ajusté }=, 128 \\
\text { Variation } \mathrm{R}^{2}=, 134^{* * *}\end{array}$} & \multicolumn{2}{|c|}{$\begin{array}{l}\mathrm{R}^{2} \text { ajusté }=, 256 \\
\text { Variation } \mathrm{R}^{2}=, 251^{* * *}\end{array}$} \\
\hline Étape $2^{b}$ & \multicolumn{2}{|c|}{$\begin{array}{l}\mathrm{R}^{2} \text { ajusté }=, 376 \\
\text { Variation } \mathrm{R}^{2}=, 082^{* * *}\end{array}$} & \multicolumn{2}{|c|}{$\begin{array}{l}\mathrm{R}^{2} \text { ajusté }=, 529 \\
\text { Variation } \mathrm{R}^{2}=, 193^{* * *}\end{array}$} & \multicolumn{2}{|c|}{$\begin{array}{l}\mathrm{R}^{2} \text { ajusté }=, 266 \\
\text { Variation } \mathrm{R}^{2}=, 145^{* * *}\end{array}$} & \multicolumn{2}{|c|}{$\begin{array}{l}\mathrm{R}^{2} \text { ajusté }=, 413 \\
\text { Variation } \mathrm{R}^{2}=, 173^{* * *}\end{array}$} \\
\hline Étape $3^{c}$ & \multicolumn{2}{|c|}{$\begin{array}{l}\mathrm{R}^{2} \text { ajusté }=, 388 \\
\text { Variation } \mathrm{R}^{2}=, 013^{* * *}\end{array}$} & \multicolumn{2}{|c|}{$\begin{array}{l}\mathrm{R}^{2} \text { ajusté }=, 546 \\
\text { Variation } \mathrm{R}^{2}=, 018^{* * *}\end{array}$} & \multicolumn{2}{|c|}{$\begin{array}{l}\mathrm{R}^{2} \text { ajusté }=, 275 \\
\text { Variation } \mathrm{R}^{2}=, 020^{* *}\end{array}$} & \multicolumn{2}{|c|}{$\begin{array}{l}\mathrm{R}^{2} \text { ajusté }=, 415 \\
\text { Variation } \mathrm{R}^{2}=\mathrm{ns}\end{array}$} \\
\hline \multirow[t]{2}{*}{ Facteurs de risque } & \multicolumn{2}{|c|}{ Garçons } & \multicolumn{2}{|c|}{ Filles } & \multicolumn{2}{|c|}{ Garçons } & \multicolumn{2}{|c|}{ Filles } \\
\hline & $-P V$ & $+\mathrm{PV}$ & $-P V$ & $+\mathrm{PV}$ & $-P V$ & $+\mathrm{PV}$ & $-P V$ & $+P V$ \\
\hline Âge & $333^{* * *}$ & $334^{* * *}$ & $271^{* * *}$ & $268 * * *$ & $\mathrm{~ns}^{\mathrm{d}}$ & ns & ns & ns \\
\hline Origine ethnique & ns & ns &, $065^{*}$ &, $068^{*}$ & ns & ns &, $079 * *$ &, $077^{*}$ \\
\hline $\begin{array}{l}\text { Ne vit pas avec ses deux parents } \\
\text { biologiques }\end{array}$ & ns & ns & ns & ns & ns & ns &, $077^{*}$ &, $076^{*}$ \\
\hline $\begin{array}{l}\text { Parents appartiennent à un groupe } \\
\text { professionnel peu qualifié }\end{array}$ &, $081^{*}$ & $078^{*}$ & ns & ns & ns & ns & ns & ns \\
\hline Adversité sans victimisation & $179 * * *$ & $167^{* * *}$ & $132 * \star *$ &, $098 * * *$ &, $200 * * *$ &, $170 * * *$ &, $199 * * *$ & $187^{* * *}$ \\
\hline
\end{tabular}


TABLEA 4 (suite)

\begin{tabular}{|c|c|c|c|c|c|c|c|c|}
\hline \multirow[t]{2}{*}{ Type de victimisation } & \multicolumn{2}{|c|}{ Garçons } & \multicolumn{2}{|c|}{ Filles } & \multicolumn{2}{|c|}{ Garçons } & \multicolumn{2}{|c|}{ Filles } \\
\hline & $-P V$ & $+\mathrm{PV}$ & $-\mathrm{PV}$ & $+\mathrm{PV}$ & $-\mathrm{PV}$ & $+\mathrm{PV}$ & $-P V$ & $+\mathrm{PV}$ \\
\hline Victimisation sexuelle &, $110^{* *}$ &, $083^{*}$ &, $224^{* * *}$ &, $181^{* * *}$ & ns & ns & $184^{* * *}$ &, $182^{* * *}$ \\
\hline $\begin{array}{l}\text { Exposition à la violence familiale } \\
\text { parentale }\end{array}$ & ns & ns & ns & ns & ns & ns &, $099^{* *}$ &, $092^{* *}$ \\
\hline $\begin{array}{l}\text { Exposition à la violence dans la commu- } \\
\text { nauté }\end{array}$ &, $120^{* *}$ &, $113^{* *}$ &, $087^{*}$ &, $083^{*}$ &, $180 * * *$ &, $177^{* *}$ & $153^{* * *}$ &, $153^{* * *}$ \\
\hline $\begin{array}{l}\text { Maltraitance commise par un parent/ } \\
\text { tuteur }\end{array}$ &, $079^{*}$ & ns &, $112^{* *}$ &, $088^{* *}$ & ns & ns &, $086^{* *}$ &, $074^{*}$ \\
\hline $\begin{array}{l}\text { Maltraitance commise par un adulte ne } \\
\text { résidant pas avec l'enfant }\end{array}$ & ns & ns &, $097^{* *}$ &, $083^{*}$ &, $097^{* *}$ &, $084^{*}$ & ns & ns \\
\hline Victimisation commise par la fratrie & ns & ns & $101^{* *}$ & $105^{* *}$ & ns & ns & ns & ns \\
\hline Victimisation commise par un pair & ns & ns &, $095^{* *}$ &, $103^{* * *}$ &, $233^{* * *}$ & $234^{* * *}$ &, $145^{* * *}$ &, $143^{* * *}$ \\
\hline $\begin{array}{l}\text { Victimisation commise par un partenaire } \\
\text { intime }\end{array}$ & $122^{* *}$ & $107^{* *}$ &, $135^{* * *}$ &, $114^{* * *}$ & ns & ns &, $123^{* * *}$ &, $117^{* * *}$ \\
\hline Polyvictimisation & &, $140 * * *$ & &, $163^{* * *}$ & &, $121^{* *}$ & & ns \\
\hline
\end{tabular}

a Incluant l'âge de l'enfant, l'origine ethnique, le groupe professionnel des parents, l'expérience d'autres types d'adversité durant l'enfance, le fait de vivre séparé d'un parent biologique.

Incluant les variables précédentes ainsi que la victimisation sexuelle, la victimisation commise par un pair, la fratrie ou un partenaire intime, la maltraitance faite par un parent/ tuteur, l'exposition à la violence familiale parentale, la maltraitance faite par un adulte ne résidant pas avec l'enfant, et l'exposition à la violence dans la communauté.

Incluant les variables précédentes ainsi que la polyvictimisation (chez les 11 à 17 ans avoir subi 13 formes de victimisation distinctes ou plus durant l'enfance et chez les 18 à 24 ans, en avoir subi 15 ou plus).

d non significatif

${ }^{*} p<0,05 ;{ }^{* *} p<0,01 ;{ }^{* * *} p<0,001$. 
d'âge ont émergé. Par exemple, l'âge était significatif uniquement pour le groupe des 11 à 17 ans, alors que le groupe ethnique l'était uniquement chez les filles. Par ailleurs, le groupe professionnel des parents constituait un prédicteur de délinquance uniquement chez les garçons âgés de 11 à 17 ans. L'impact de la victimisation sur la délinquance variait selon le type de victimisation, le sexe et le groupe d'âge. La victimisation sexuelle durant l'enfance était fortement associée à la délinquance des filles, mais elle l'était moins chez les garçons de moins de 18 ans. L'exposition à la violence dans la communauté, ainsi que la victimisation par les pairs, étaient des facteurs plus significatifs sur la délinquance juvénile rapportée par les jeunes adultes et produisaient des effets différents chez les garçons et les filles. La maltraitance commise par un parent ou un tuteur, comme la victimisation sexuelle, entraînait des conséquences plus grandes sur la délinquance des filles que celles des garçons. La victimisation perpétrée par un partenaire intime influençait les cotes de délinquance des moins de 18 ans chez les deux sexes et celles du groupe de femmes de plus de 18 ans, mais n'était pas significativement associée à la délinquance juvénile rapportée par les hommes de plus de 18 ans. Deux types de victimisation étaient, encore une fois, uniquement significatifs chez les filles: la victimisation commise par la fratrie pour celles âgées de 11 à 17 ans et l'exposition à la violence familiale parentale pour celles de 18 à 24 ans. La polyvictimisation avait un impact significatif sur la délinquance, tant chez les garçons que chez les filles de 11 à 17 ans. Chez le groupe plus âgé (18 à 24 ans), la polyvictimisation avait un impact moins significatif sur la délinquance juvénile des hommes et aucun impact sur celle des femmes.

\section{Discussion}

Les conclusions tirées de la présente étude basée sur une population au Royaume-Uni appuient certaines des conclusions de l'étude américaine, réalisée par Finkelhor et al. (2007a, 2007b, 2009b), sur la polyvictimisation des jeunes et ses impacts. L'âge et le sexe (masculin) se sont avérés des facteurs de risque significatifs pour la polyvictimisation au Royaume-Uni, tout comme aux États-Unis, bien qu'il y ait de nettes différences selon le sexe par rapport aux types de victimisation rapportés par les garçons et les filles de différents âges et de leur impact sur la délinquance. L'exposition à la violence dans la communauté, la vic- 
timisation par les pairs et la maltraitance perpétrée par un parent/tuteur augmentent significativement les probabilités qu'un jeune ou un jeune adulte devienne une polyvictime. Ces résultats corroborent l'idée selon laquelle les trajectoires de polyvictimisation incluent le fait de résider dans une communauté dangereuse, de vivre dans un milieu familial dangereux ou d'avoir des problèmes émotionnels durant l'enfance. Nous supposons que ces facteurs ont une influence sur la polyvictimisation sur la base des travaux de Finkelhor et al. (2007a, 2007b, 2009b). Cependant, la présente recherche est basée sur une étude transversale, contrairement à l'étude à trois mesures répétées réalisée par l'équipe des États-Unis (Finkelhor et al., 2007a. 2007b, 2009b), ce qui limite la portée des conclusions possibles. De plus, dans la présente étude, les données disponibles au sujet de la quatrième trajectoire de polyvictimisation, soit le fait de vivre au sein d'une famille chaotique avec des problèmes multiples, sont limitées. Dans le cadre de cet article, il a été impossible d'évaluer si le fait d'habiter dans ce genre de famille mène à la polyvictimisation, bien que nous ayons constaté que les hauts niveaux d'adversité durant l'enfance étaient particulièrement significatifs. L'incapacité était aussi un facteur de risque de polyvictimisation chez les jeunes adultes, ceux-ci ayant un risque de polyvictimisation trois fois supérieur à ceux qui ne souffraient pas d'incapacité. Une publication récente, basée sur cette étude, a conclu que la polyvictimisation avait un impact particulièrement significatif sur le bien-être émotionnel des enfants, des jeunes et des jeunes adultes (Radford et al., 2013). Or, bien que ces conclusions nous éclairent quant aux trajectoires de polyvictimisation au Royaume-Uni, il est évident que davantage de recherches, surtout longitudinales, devront être réalisées afin de confirmer les trajectoires suggérées dans la littérature (Finkelhor et al., 2007a, 2007b, 2009b).

Des différences selon le sexe ont été observées entre la délinquance, la victimisation et la polyvictimisation, avec un impact particulièrement frappant de la victimisation sexuelle sur la délinquance des filles. Cette constatation appuie l'hypothèse de Wilson et Spatz-Widom (2008), selon laquelle les filles agressées et exploitées sexuellement sont plus enclines à être criminalisées que les filles non agressées. La polyvictimisation avait un plus grand impact sur la délinquance juvénile des jeunes de moins de 18 ans que chez les plus âgés, son impact étant significativement réduit chez les hommes et nul chez les femmes de 18 à 24 ans. Une explication possible serait que les jeunes polyvictimisés 
sont plus portés à s'engager dans des actes délinquants à un plus jeune âge, mais pas nécessairement plus tard.

\section{Limites}

La nature des données obtenues représente une limite importante de l'étude. Alors que les autres études visant à identifier des trajectoires de polyvictimisation sont basées sur plusieurs cycles de collecte de données, la présente recherche est transversale. Par conséquent, quelques hypothèses ont été lancées au sujet de la relation de cause à effet entre les facteurs de risque et la polyvictimisation. Ceci souligne, tel qu'il a été précisé précédemment, le besoin de recherches subséquentes sur la polyvictimisation.

\section{Implications}

De nombreuses répercussions sur la pratique découlent des conclusions de cette étude. Dans le but de prévenir la criminalisation précoce des enfants victimisés et polyvictimisés, il est essentiel que les enfants les plus vulnérables soient repérés rapidement; qu'une évaluation adéquate de leurs besoins soit effectuée; et que des services soient mis en place afin d'intervenir et de corriger les conséquences néfastes liées au fait de subir de la violence et de la maltraitance à la maison, à l'école et dans la communauté. Les professionnels qui travaillent auprès des enfants et des jeunes doivent être vigilants à l'égard des aspects concomitants et cumulatifs de la victimisation des jeunes, particulièrement la maltraitance, la victimisation sexuelle et la victimisation commise par les pairs. Cela signifie que si un enfant dans un milieu donné, comme l'école, devient la cible d'intimidation par ses pairs, ou bien s'avère un intimidateur pour les autres, les professionnels doivent être équipés des connaissances, des compétences, de la sensibilité et des ressources nécessaires pour déceler d'autres types de victimisation qui pourraient avoir lieu dans d'autres sphères de la vie de l'enfant. Les experts en délinquance juvénile, en protection de l'enfance, en éducation et en services d'aide sociale à l'enfance particulièrement, pourraient travailler en collaboration afin d'améliorer l'identification et les réponses promptes destinées aux enfants et aux jeunes victimisés et polyvictimisés. 


\section{Références}

Andrews, G., Corry, J., Slade, T., Issakidis, C., \& Swanston, H. (2004). Child sexual abuse. Comparative quantification of health risks. Geneva: World Health Organisation.

Arata, C. M., Langhinrichsen-Rohling, J., Bowers, D., \& O’Brien, N. (2007). Differential correlates of multi-type maltreatment among urban youth. Child Abuse and Neglect, 31 (4), 393-415.

Bensley, L. S., Van Eenwyk, J., Spieker, S. J., \& Schoder, J. (1999). Self-reported abuse history and adolescent problem behaviour. I. Antisocial and suicidal behaviour. Journal of Adolescent Health, 24, 163-172.

Briere, J. (1996). Trauma Symptom Checklist for Children (TSCC) professional manual. Odessa, FL: Psychological Assessment Resources.

Briere, J., \& Runtz, M. (1989). The Trauma Symptom Checklist (TSC-33): Early data on a new scale. Journal of Interpersonal Violence, 4 (2), 151-163.

Burton, D., Duty, K., \& Leibowitz, G. (2011) Differences between Sexually Victimized and Nonsexually Victimized Male Adolescent Sexual Abusers: Developmental Antecedents and Behavioral Comparisons. Journal of Child Sexual Abuse 20 (1), 77-93.

Cawson, P., Wattam, C., Brooker, S., \& Kelly, G. (2000). Child maltreatment in the United Kingdom. London: NSPCC.

Chaplin, R., Flatley. J., \& Smith, K. (2011). Crime in England and Wales 2010-11: Findings from the British Crime Survey and Police Recorded Crime (2nd ed). London: Home Office.

Cuevas, C. A., Finkelhor, D., Turner, H. A. \& Ormrod, R. K. (2007). Juvenile delinquency and victimization: A theoretical typology. Journal of Interpersonal Violence, 22 (12), 1581-1602.

Department for Children, Schools and Families. (2010). Working together to safeguard children: A guide to inter-agency working to safeguard and promote the welfare of children. Repéré à https://www.education.gov.uk/ publications/standard/publicationDetail/Page1/DCSF-00305-2010.

Donga, M., Andaa, R., Felitti, V., Dubea, S., Williamson, D., Thompson, T., Loo, C., \& Giles, W. (2004). The interrelatedness of multiple forms of childhood abuse, neglect, and household dysfunction. Child Abuse $\mathfrak{F}$ Neglect, 28, 771-784.

Edwards, V. J., Holden, G. W., Felitti, V. J., \& Anda, R. F. (2003). Relationship between multiple forms of childhood maltreatment and adult mental health in community respondents: results from the adverse childhood experiences study. The American Journal of Psychiatry, 160 (8), 1453-1460.

Egeland, B., Yates, T., Appleyard, K., \& van Dulmen, M. (2002). The long-term consequences of maltreatment in the early years: A developmental pathway model to antisocial behavior. Children's Services: Social Policy, Research, and Practice, 5, 249-260.

Farrington, D., Coid, J., Harnett, L., Joliffe, D., Soteriou, N., Turner, R., \& West, D. (2006). Criminal Careers up to Age 50 and Life Success Up to Age 48: New Findings from the Cambridge Study in Delinquent Development. London: Home Office. 
Finkelhor, D., Ormrod, R. K., Turner, H. A., \& Hamby, S. L. (2005). The victimization of children and youth : a comprehensive, national survey. Child Maltreatment, 10 (1), 5-25.

Finkelhor, D., Ormrod, R. K., \& Turner, H.A. (2007a). Poly-victimization: A neglected component in child victimization. Child Abuse and Neglect, 31 (1), 7-26.

Finkelhor, D., Ormrod, R. K., \& Turner, H.A. (2007b). Polyvictimization and trauma in a national longitudinal cohort. Developmental Psychopathology, 19 (1), 149-166.

Finkelhor, D., Turner, H., Ormrod, R., \& Hamby, S.L. (2009a). Violence, abuse, and crime exposure in a national sample of children and youth. Pediatrics, 124 (5), 1411-1423.

Finkelhor, D., Ormrod, R. K., \& Turner, H. A. (2009b). Lifetime assessment of poly-victimization in a national sample of children and youth. Child Abuse and Neglect, 33 (7), 403-411.

Gilbert, R., Spatz Widom, C., Browne, K., Fergusson, D., Webb, E., \& Janson, S. (2009). Burden and consequences of child maltreatment in high-income countries. The Lancet, 373 (9657), 68-81. doi: 10.1016/S0140-6736 (08) 61706-7.

Green, S., Russo, M., Navratil, J., \& Loeber, R. (1999). Sexual and physical abuse among adolescent girls with disruptive behaviour problems. Journal of Child and Family Studies, 8, 151-168.

Gustafsson, P. E., Nilsson, D., \& Svedin, C. G. (2009). Polytraumatization and psychological symptoms in children and adolescents. European Child and Adolescent Psychiatry, 18 (5), 274-283.

Hamby, S. L., Finkelhor, D., Ormrod, R. K., \& Turner, H. A. (2004a). The comprehensive Juvenile Victimization Questionnaire. Durham, $\mathrm{NH}$ : University of New Hampshire.

Hamby, S. L., Finkelhor, D., Ormrod, R. K., \& Turner, H. A. (2004b). The Juvenile Victimization Questionnaire (JVQ): administration and scoring manual. Durham, NH: Crimes Against Children Research Center.

Kinard, E. M. (2004). Methodological issues in assessing the effects of maltreatment characteristics on behavioral adjustment in maltreated children. Journal of Family Violence, 19 (5), 303-318.

Moffitt, T. E., Caspi, A., Harrington, H., \& Milne, B. J. (2002). Males on the life-course-persistent and adolescence-limited, antisocial pathways: Followup at age 26 years. Development and Psychopathology, 14 (1), 179-207.

Pinheiro, P. (2006). World report on violence against children. Geneva: United Nations.

Radford, L., Corral, S., Bradley, C., Fisher, H., Collishaw, S., Bassett, C. \& Howat, N. (2011). Child Abuse and Neglect in the UK Today. London : NSPCC. Repéré à www.nspcc.org.uk/childstudy

Radford, L., Corral, S., Bradley, C., \& Fisher, H. (2013). The prevalence and impact of child maltreatment and other types of victimization in the UK: Findings from a population survey of caregivers, children and young people 
and young adults. Child Abuse and Neglect, 37 (10), 801-813. Repéré à http:// dx.doi.org/10.1016/j.chiabu.2013.02.004

Radford, L., \& J. Ellis. (2012). Offering children confidentiality in research: what are the limits? In J.Fleming \& T. Boeck (eds.), Involving children and young people in bealth and social care research (118-126). London: Routledge.

Spatz-Widom, C., \& Raskin-White, H. (1997). Problem behaviours in abused and neglected children grown up: prevalence and co-occurrence of substance abuse, crime and violence. Criminal Behaviour and Mental Health, 7, 287-310.

Turner, H. A., Finkelhor, D., \& Ormrod, R. K. (2006). The effect of lifetime victimization on the mental health of children and adolescents. Social Science and Medicine, 62 (1), 13-27.

Wilson, H. \& Spatz Widom, C. (2008). An examination of risky sexual behavior and HIV in victims of child abuse and neglect: A 30-year follow-up. Health Psychology, 27 (2), 149-158.

Wolfe, D. A., Scott, K., Wekerle, C., \& Pittman., A. (2001). Child maltreatment: Risk of adjustment problems and dating violence in adolescence. Journal of the American Academy of Child $\mathfrak{F}$ Adolescent Psychiatry, 40 (3), 282-289.

Wong, T., Slotboom, A., \& Bijleveld, C. (2010). Risk factors for delinquency in adolescent and young adult females: A European review. European Journal of Criminology, 7 (4), 266-284.

ABSTRACT - This paper extends work on the impact and nature of cumulative victimization on children's, adolescents' and young adults' self-reported deviancy. As part of a representative UK household survey conducted in 2009, exposure to a wide range of victimization events and self-reported delinquency was assessed using 4036 interviews with participants from two age groups: children aged between 11 and 17 and young adults aged 18 to 24 years. Age, gender (being male), and experiencing certain types of victimization significantly increased the odds of experiencing lifetime childhood polyvictimization. The impact of victimization on delinquency varied by victimization type, gender and age group. Experiencing sexual victimization in childhood had a strong association with delinquency for females but had less impact on males under the age of 18. Polyvictimization had the greatest impact on delinquency among children aged 11 to 17 but it was not significant for young adult females. Professionals who work with children and young people need to be alert to the overlapping and cumulative aspects of child victimization and equipped to identify and respond to those vulnerable to being polyvictimized.

KEY WORDS - Polyvictimization, delinquency, childhood victimization, child maltreatment, United Kingdom.

RESUMEN - El presente estudio explora el impacto y la naturaleza de la acumulación de victimizaciones sobre la desviación autodeclarada de los niños, de los adolescentes y de los jóvenes adultos. En el marco de una estudio representativo llevado a cabo en 2009 en los hogares del Reino Unido, la exposición a una amplia gama de experiencias de victimización y de delincuencia autodeclarada han sido evaluadas gracias a 4.036 entrevistas a los participantes de dos grupos: niños entre 11 y 17 años de edad y 
jóvenes adultos cuyas edades estaban comprendidas entre los 18 y los 24 años. La edad, el sexo (masculino) y el hecho de haber vivido ciertas formas de victimización aumentaban considerablemente las probabilidades de victimización durante la infancia. El impacto de la victimización sobre la delincuencia variaba según el tipo de victimización, el sexo y el grupo etareo. La victimización sexual durante la infancia estaba fuertemente asociada a la delincuencia femenina, pero tenia menos impacto en los varones menores de 18 años. La polivictimización tenia mas impacto en los niños de entre 11 y 17 años, pero no influenciaba significativamente aquélla de las mujeres adultas. Los profesionales que trabajan con niños y jóvenes deben estar al tanto de las concomitancias y del carácter cumulativo de la victimización en la infancia. También deben estar formados para identificar y ayudar aquellos que son vulnerables a la polivictimizacion.

PALABRAS-Clave - Polivictimización, delincuencia en la infancia, maltrato, Reino Unido. 